\title{
A COMPARISON OF NTU VALUES IN A COOPERATIVE GAME WITH INCOMPLETE INFORMATION
}

\author{
Andrés Salamanca \\ University of Southern Denmark, Denmark \\ salamanca@sam.sdu.dk
}

\begin{abstract}
Several "value-like" solution concepts are computed and compared in a cooperative game with incomplete information and non-transferable utility. We will show that the difference between these values is caused by how payoff strategic possibilities of coalitions of the game are handled.

Keywords: Cooperative games, incomplete information, non-transferable utility.

JEL Classification Numbers: C71, D82.
\end{abstract}

\section{INTRODUCTION}

B y introducing the concept of "virtual utility", Myerson (1984) proposed B a general notion of value for cooperative games with incomplete information. The so-called M-value generalizes the Shapley non-transferable utility (NTU) value. ${ }^{1}$ The pertinence of the M-value was tested afterwards by de Clippel (2005) in an eloquent three-player game. Later, building on Myerson's virtual utility approach, Salamanca (2019) defined an alternative value

This short paper is built upon some results from my master theses in Economics (at Toulouse School of Economics) and Applied Mathematics (at the Universidad Nacional de Colombia). I would like to thank Françoise Forges, Francisco Lozano, Michel Le Breton, Jérôme Renault, and Geoffroy de Clippel for helpful comments and discussion. This paper also benefited from the valuable comments of the editor and an anonymous referee. Any errors are, of course, my own responsibility.

1 The Shapley NTU value is sometimes referred to as the $\lambda$-transfer value.

Copyright (c) Andrés Salamanca / 4(1), 2019, 109-117. 
concept called the S-value, which generalizes the Harsanyi NTU value. Both the M-value and the S-value reflect not only the signaling costs associated with incentive compatibility, but also the fact that individuals negotiate at the interim stage (i.e., after each player has received his private information). Salamanca (2019) also showed that the M-value differs from the S-value in that the former is less sensitive to some informational externalities. In this short paper we analyze a simple example of an NTU game in which these two solution concepts differ because of the way payoff strategic possibilities of subcoalitions are handled. ${ }^{2}$ We also study our example under the assumption of ex-ante negotiation. In that situation, the players make coalitional agreements before they acquire their private information.

\section{COOPERATIVE GAMES WITH INCOMPLETE INFORMATION}

The model of a cooperative game with incomplete information is as follows. Let $N=\{1,2, \ldots, n\}$ denote the set of players. For each (non-empty) coalition $S \subseteq N, D_{S}$ denotes the (finite) set of joint decisions for $S$. The sets of joint decisions are assumed to be superadditive, that is, for any two disjoint coalitions $S$ and $R, D_{R} \times D_{S} \subseteq D_{R \cup S}$. For any player $i \in N$, we let $T_{i}$ denote the (finite) set of possible types of player $i$. The interpretation is that $t_{i}$ represents the private information possessed by player $i$. We use the notations $t_{S}=\left(t_{i}\right)_{i \in S} \in T_{S}=\prod_{i \in S} T_{i}, t_{-i}=t_{N \backslash i} \in T_{-i}=T_{N \backslash i}$. For simplicity we drop the subscript $N$ in the case of the grand coalition, so we define $D:=D_{N}$ and $T:=T_{N}$. We assume that types are randomly chosen according to a common prior probability distribution $p$ defined on $T$. The utility function of player $i \in N$ is defined to be $u_{i}: D \times T \rightarrow \mathbb{R}$. As in most of the literature in cooperative game theory, we assume that coalitions are orthogonal, namely, when coalition $S$ chooses an action which is feasible for it, the payoffs to the members of $S$ do not depend on the actions of the complementary coalition $N \backslash S$.

A mechanism for coalition $S \subseteq N$ is a pair of functions $\left(\mu_{S}, x_{S}\right)$ defined by: ${ }^{3}$

$$
\begin{aligned}
\mu_{S}: T & \rightarrow \Delta\left(D_{S}\right) & x_{S}: T & \rightarrow \mathbb{R}_{-}^{S} \\
t & \mapsto \mu_{S}(\cdot \mid t) & t & \mapsto\left(x_{S}^{i}(t)\right)_{i \in S}
\end{aligned}
$$

2 Our example is reminiscent of an NTU game with complete information proposed by Roth (1980).

3 This definition is adapted from the mechanisms with sidepayments considered by Myerson (2007). For any finite set $A, \Delta(A)$ denotes the set of probability distributions over $A$. 
Both mappings, $\mu_{S}$ and $x_{S}$, are measurable w.r.t. the information of the members of $S$. The component $\mu_{S}$ is a type-contingent lottery on the set of feasible decisions for $S$, while $x_{S}$ is a vector of type-contingent utility decrements (free disposal). The mechanism $\left(\mu_{S}, x_{S}\right)(S \neq N)$ stands as a threat to be carried out only if $N \backslash S$ refuses to cooperate with $S$. We denote by $\mathcal{F}_{S}$ the set of mechanisms for coalition $S$.

\section{THE EXAMPLE}

Let $r$ be a parameter with $0<r<1 / 2$. For each value of $r$, we consider the following cooperative game with incomplete information: The set of players is $N=\{1,2,3\}$. Player 1 has private information about one of two possible states, $T=\{H, L\}$, which happen with prior probabilities $p(H)=1-p(L)=4 / 5$. Feasible decisions for coalitions are $D_{\{i\}}=\left\{d_{i}\right\}(i \in N), D_{\{i, j\}}=\left\{\left[d_{i}, d_{i}\right], d_{i j}\right\}$ $(i \neq j), D_{N}=\left\{\left[d_{1}, d_{2}, d_{3}\right],\left[d_{12}, d_{3}\right],\left[d_{13}, d_{2}\right],\left[d_{23}, d_{1}\right]\right\}$. Utility functions are given by:

\begin{tabular}{c|cc}
$\left(u_{1}, u_{2}, u_{3}\right)$ & $H$ & $L$ \\
\hline$\left[d_{1}, d_{2}, d_{3}\right]$ & $(0,0,0)$ & $(0,0,0)$ \\
{$\left[d_{12}, d_{3}\right]$} & $(50,50,0)$ & $(40,40,0)$ \\
{$\left[d_{13}, d_{2}\right]$} & $(100 r, 0,100(1-r))$ & $(40 r, 0,40(2-r))$ \\
{$\left[d_{23}, d_{1}\right]$} & $(0,100 r, 100(1-r))$ & $(0,40 r, 40(2-r))$
\end{tabular}

Feasible decisions are understood as follows: Decision $d_{i}$ denotes player $i$ 's non-cooperative option, which leaves her with her reservation utility normalized to zero. When coalition $\{i, j\}$ forms and its members agree on an outcome $d \in D_{\{i, j\}}$, player $k$ (in the complementary coalition) is left alone with the only possibility to choose $d_{k}$. Hence, $\left[d_{1}, d_{2}, d_{3}\right]$ denotes the outcome in which no player cooperates, and $\left[d_{i j}, d_{k}\right]$ corresponds to the cooperative outcome in which players $i$ and $j$ form a coalition and share the proceeds of cooperation as specified above. No other outcomes are possible.

Player 3 can be considered as weak in the sense that she can only offer players 1 and 2 a payoff that is strictly lower than what they can both obtain by acting together in coalition $\{1,2\}$. Then it does appear that coalitions $\{1,3\}$ and $\{2,3\}$ are less likely to form than $\{1,2\}$. Moreover, the smaller $r$ is, the less utility player 3 can transfer to players 1 and 2 , and therefore the less likely it should be that $\{1,3\}$ or $\{2,3\}$ form. 
In this game, efficient allocations can be made (Bayesian) incentive compatible. We shall thus assume that all information is public at the implementation stage, which implies that any mechanism in $\mathcal{F}_{S}$ can be enforced once it is agreed upon. ${ }^{4}$ As a result, virtual utility specializes to a rescaling of actual utility and one obtains simple expressions for both the M-value and the S-value.

\section{CONTRACTING AT THE INTERIM STAGE}

At the interim stage each player knows her own type $t_{i} \in T_{i}$, and hence, we let $p_{i}\left(t_{-i} \mid t_{i}\right)$ denote the conditional probability of $t_{-i}$ that player $i$ infers given her type $t_{i}$.

For a given coalition $S$, we write $u_{i}\left(\mu_{S}, t\right)$ for the linear extension of the utility functions over $\mu_{S}\left(\cdot \mid t_{S}\right) .{ }^{5}$ We define $u_{i}\left(\left(\mu_{S}, x_{S}\right), t\right):=u_{i}\left(\mu_{S}, t\right)+x_{S}^{i}\left(t_{S}\right)$ to be player $i$ 's expected utility from $\left(\mu_{S}, x_{S}\right)$ conditional on state $t$. Hence, $U_{i}\left(\mu_{S}, x_{S} \mid t_{i}\right):=\sum_{t_{-i} \in T_{-i}} p_{i}\left(t_{-i} \mid t_{i}\right) u_{i}\left(\left(\mu_{S}, x_{S}\right), t\right)$ denotes $i$ 's interim expected utility from $\left(\mu_{S}, x_{S}\right)$ given her type $t_{i}$. A mechanism $\left(\bar{\mu}_{N}, \bar{x}_{N}\right)$ is (interim) efficient for the grand coalition if there exists a non-negative vector $\lambda=$ $\left(\lambda_{i}\left(t_{i}\right)\right)_{i \in N, t_{i} \in T_{i}}$, such that $\left(\bar{\mu}_{N}, \bar{x}_{N}\right)$ maximizes the social welfare function

$$
\sum_{i \in N} \sum_{t_{i} \in T_{i}} \lambda_{i}\left(t_{i}\right) U_{i}\left(\mu_{N}, x_{N} \mid t_{i}\right)
$$

Thus, $\lambda$ is normal to the interim Pareto frontier at the utility allocation implemented by $\left(\bar{\mu}_{N}, \bar{x}_{N}\right)$.

If we fix a vector $\lambda$ of utility weights as above, then given a coalition $S$ and a mechanism $\left(\mu_{S}, x_{S}\right)$, the virtual utility of player $i$ in state $t$ is defined as

$$
v_{i}^{\lambda}\left(\left(\mu_{S}, x_{S}\right), t\right):=\frac{\lambda_{i}\left(t_{i}\right)}{p_{i}\left(t_{i}\right)} u_{1}\left(\left(\mu_{S}, x_{S}\right), t\right),
$$

where $p_{i}\left(t_{i}\right)$ is $i$ 's marginal probability of her type $t_{i}$.

Consider the fictitious game in which, conditionally on every state $t$, virtual utilities are transferable. The worth of coalition $S \subseteq N$ in state $t \in T$, when its members agree on the mechanism $\left(\mu_{S}, x_{S}\right)$, is defined to be

$$
W_{S}^{\lambda}\left(\left(\mu_{S}, x_{S}\right), t\right):=\sum_{i \in S} v_{i}^{\lambda}\left(\left(\mu_{S}, x_{S}\right), t\right)
$$

4 Here, the only issue is the revelation of private information at the negotiation stage.

5 Since coalitions are orthogonal $u_{i}\left(\mu_{S}, t\right)$ is well defined. 
For a given profile of threats, $\eta=\left(\left(\mu_{S}, x_{S}\right)\right)_{S \subseteq N}, W^{\lambda}(\eta, t):=\left(W_{S}^{\lambda}\left(\left(\mu_{S}, x_{S}\right), t\right)\right)_{S \subseteq N}$ defines a TU game in state $t$. Let $\phi_{i}\left(W^{\lambda}(\eta, t)\right)$ denote the Shapley TU value of player $i$ in the game $W^{\lambda}(\eta, t)$. A mechanism $\left(\bar{\mu}_{N}, \bar{x}_{N}\right)$ for the grand coalition is (virtually) equitable if for each $t_{i} \in T_{i}$ of every $i \in N$,

$$
\sum_{t_{-i} \in T_{-i}} p_{i}\left(t_{-i} \mid t_{i}\right) v_{i}^{\lambda}\left(\left(\bar{\mu}_{N}, \bar{x}_{N}\right), t\right)=\sum_{t_{-i} \in T_{-i}} p_{i}\left(t_{-i} \mid t_{i}\right) \phi_{i}\left(W^{\lambda}(\eta, t)\right) .
$$

That is, an equitable mechanism $\left(\bar{\mu}_{N}, \bar{x}_{N}\right)$ gives every type of a player her (conditionally) expected Shapley TU value in the fictitious game.

A mechanism $\left(\bar{\mu}_{N}, \bar{x}_{N}\right)$ is called a bargaining solution if there exists a strictly positive vector ${ }^{6} \lambda$ and a vector of threats $\eta=\left(\mu_{S}, x_{S}\right)_{S \subseteq N}$ such that $\left(\bar{\mu}_{N}, \bar{x}_{N}\right)$ is efficient and equitable given $(\lambda, \eta)$. Different NTU values can be defined depending on how $\eta$ is determined.

Definition 1 (M-Value). A bargaining solution $\left(\bar{\mu}_{N}, \bar{x}_{N}\right)$ supported by $\lambda$ and $\bar{\eta}=\left(\bar{\mu}_{S}, \bar{x}_{S}\right)_{S \subseteq N}$ is called an M-solution iff for every coalition $S \neq N,\left(\bar{\mu}_{S}, \bar{x}_{S}\right)$ solves

$$
\max _{\left(\mu_{S}, x_{S}\right) \in \mathcal{F}_{S}} \sum_{t \in T} p(t) W_{S}^{\lambda}\left(\left(\mu_{S}, x_{S}\right), t\right) .
$$

The interim utility allocation generated by $\left(\bar{\mu}_{N}, \bar{x}_{N}\right)$ is called an M-value.

By solving (2), the M-value measures the strength of coalitions as the maximum joint gains that can be allocated inside the coalition. However, it disregards the restrictions the players face when sharing such gains.

Claim 1 (M-value). For any given $r \in(0,1 / 2)$, the unique M-value of our example is the interim utility allocation

$$
\left(U_{1}^{H}, U_{1}^{L}, U_{2}, U_{3}\right)=\left(\frac{100}{3}, \frac{80}{3}, 32,32\right) .
$$

Proof. The interim Pareto frontier coincides with the hyperplane $\frac{4}{5} U_{1}^{H}+\frac{1}{5} U_{1}^{L}+$ $U_{2}+U_{3}=96$ on the individually rational zone. Thus, (3) is efficient. Since bargaining solutions are individually rational, an M-value can only be supported by the utility weights $\left(\lambda_{1}^{H}, \lambda_{1}^{L}, \lambda_{2}, \lambda_{3}\right)=(4 / 5,1 / 5,1,1)$. Hence, virtual and real utilities coincide. After computation of threats according to (2), equations in (1) yield (3).

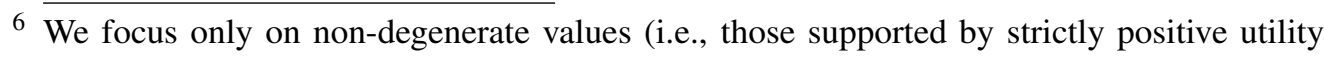
weights). 
The M-value prescribes the same allocation regardless of the value of $r$. Furthermore, it treats all players symmetrically. This is due to the fact that, by computing threats according to (2), we proceed as if coalitions $\{1,3\}$ and $\{2,3\}$ could agree on an equitable distribution of the total gains, something that is not possible in the original NTU game. Thus, we may argue that threats in the M-value are not "credible".

A mechanism $\left(\mu_{S}, x_{S}\right)$ is called egalitarian for $S$ w.r.t. $\lambda$ and $\left(\mu_{S \backslash i}, x_{S \backslash i}\right)_{i \in S}$ iff for each $t_{i} \in T_{i}$ of every $i \in S$,

$$
\begin{aligned}
\sum_{t_{-i} \in T_{-i}} p_{i}\left(t_{-i} \mid t_{i}\right) & \sum_{j \in S \backslash i}\left[v_{i}^{\lambda}\left(\left(\mu_{S}, x_{S}\right), t\right)-v_{i}^{\lambda}\left(\left(\mu_{S \backslash j}, x_{S \backslash j}\right), t\right)\right] \\
& =\sum_{t_{-i} \in T_{-i}} p_{i}\left(t_{-i} \mid t_{i}\right) \sum_{j \in S \backslash i}\left[v_{j}^{\lambda}\left(\left(\mu_{S}, x_{S}\right), t\right)-v_{j}^{\lambda}\left(\left(\mu_{S \backslash i}, x_{S \backslash i}\right), t\right)\right] .
\end{aligned}
$$

That is, a mechanism $\left(\mu_{S}, x_{S}\right)$ is egalitarian if the expected average virtual contribution of the different players in $S$ to player $i$ equals the expected average virtual contribution of player $i$ to the different players in $S$ as assessed by type $t_{i}$.

For the example under consideration, a mechanism $\left(\mu_{S}, x_{S}\right)$ is egalitarian for coalition $S=\{1, j\}(j=2,3)$ if

$$
v_{1}^{\lambda}\left(\left(\mu_{S}, x_{S}\right), t\right)=v_{j}^{\lambda}\left(\left(\mu_{S}, x_{S}\right), t\right), \quad \forall t \in T .
$$

Similarly, a mechanism $\left(\mu_{S}, x_{S}\right)$ is egalitarian for coalition $S=\{2,3\}$ if

$$
\sum_{t \in T} p(t) v_{2}^{\lambda}\left(\left(\mu_{S}, x_{S}\right), t\right)=\sum_{t \in T} p(t) v_{3}^{\lambda}\left(\left(\mu_{S}, x_{S}\right), t\right) .
$$

Because players 2 and 3 cannot make an agreement contingent on player 1 's private information, the egalitarian equality for coalition $\{2,3\}$ holds only in expectation.

Definition 2 (S-Value). A bargaining solution $\left(\bar{\mu}_{N}, \bar{x}_{N}\right)$ supported by $\lambda$ and $\bar{\eta}=\left(\bar{\mu}_{S}, \bar{x}_{S}\right)_{S \subseteq N}$ is called an $S$-solution iff for every coalition $S \neq N,\left(\bar{\mu}_{S}, \bar{x}_{S}\right)$ solves

$$
\begin{aligned}
& \max _{\left(\mu_{S}, x_{S}\right) \in \mathcal{F}_{S}} \sum_{t \in T} p(t) W_{S}^{\lambda}\left(\left(\mu_{S}, x_{S}\right), t\right) \\
& \text { s.t. }(4) \text { w.r.t. } \lambda \text { and }\left(\bar{\mu}_{S \backslash i}, \bar{x}_{S \backslash i}\right)_{i \in S}
\end{aligned}
$$

The interim utility allocation generated by $\left(\bar{\mu}_{N}, \bar{x}_{N}\right)$ is called an $S$-value. 
By imposing the egalitarian constraints, the S-value takes into account the equity restrictions that coalitions face when sharing the proceeds of cooperation.

Claim 2 (S-value). For a given $r \in(0,1 / 2)$, the unique $S$-value of our example is the interim utility allocation

$$
\begin{aligned}
\left(U_{1}^{H}, U_{1}^{L}, U_{2}, U_{3}\right) & \\
= & \left(50-\frac{100}{3} r\left(\frac{88-88 r}{96-88 r}\right), 40-\frac{80}{3} r\left(\frac{88-44 r}{96-88 r}\right), 48-\frac{88}{3} r, \frac{176}{3} r\right) .
\end{aligned}
$$

Proof. The same reasoning as in the proof of Claim 1.

The S-value gives less to player 3 compared to the $\mathrm{M}$-value. This is due to the fact that two-person coalitions with player 3 cannot fully distribute the total gains from cooperation in an equitable way. This lack of transferability increases as long as $r$ decreases to 0 , which explains why the $S$-value converges to the allocation $(50,40,48,0)$ as $r$ vanishes. It seems that the S-value reflects the power structure of this game better than the $\mathrm{M}$-value, in particular for a small $r$.

\section{CONTRACTING AT THE EX-ANTE STAGE}

When contracting takes place at the ex-ante stage, players face a cooperative game under incomplete information but with symmetric uncertainty. Then we may apply both the Shapley NTU value and the Harsanyi NTU value to the associated characteristic function of the game.

We let $U_{i}\left(\mu_{S}, x_{S}\right):=\sum_{t \in T} p(t) u_{i}\left(\left(\mu_{S}, x_{S}\right), t\right)$ denote $i$ 's ex-ante expected utility from $\left(\mu_{S}, x_{S}\right)$. The set of feasible payoff allocations for coalition $S \subseteq N$ is given by $V(S)=\left\{\left(U_{i}\left(\mu_{S}, x_{S}\right)\right)_{i \in S} \mid\left(\mu_{S}, x_{S}\right) \in \mathcal{F}_{S}\right\}$. Then the ex-ante characteristic function of our example is:

$$
\begin{aligned}
V_{r}(\{i\}) & =\left\{u_{i} \mid u_{i} \leq 0\right\}, \quad \forall i \in N, \\
V_{r}(\{1,2\}) & =\left\{\left(u_{1}, u_{2}\right) \mid u_{1} \leq 48, u_{2} \leq 48\right\}, \\
V_{r}(\{i, 3\}) & =\left\{\left(u_{i}, u_{3}\right) \mid u_{i} \leq 88 r, u_{3} \leq 96-88 r\right\},(i=1,2), \\
V_{r}(N) & =\operatorname{comp}\left(\left\{u_{12}, u_{13}, u_{23}\right\}\right),
\end{aligned}
$$

where $u_{13}:=(88 r, 0,96-88 r), u_{23}:=(0,88 r, 96-88 r), u_{12}:=(48,48,0)$ and, for any finite set $A, \operatorname{comp}(A)$ denotes the closed comprehensive hull of $A$. 
Definition 3 (Shapley NTU value). A payoff configuration $u=\left(u_{S}\right)_{S \subseteq N}$, with $u_{S} \in V(S)$, is a Shapley NTU solution of a game $(V, N)$ if there exists a vector of strictly positive utility weights $\lambda=\left(\lambda_{i}\right)_{i \in N}$ such that:

$$
\begin{gathered}
\sum_{i \in S} \lambda_{i} u_{S}^{i} \geq \sum_{i \in S} \lambda_{i} v^{i}, \quad \forall v \in V(S), \\
\lambda_{i} u_{N}^{i}=\phi_{i}\left(w^{\lambda}\right), \quad \forall i \in N,
\end{gathered}
$$

where $w^{\lambda}$ is the TU game defined by $w^{\lambda}(S)=\sum_{i \in S} \lambda_{i} u_{S}^{i}$, for every $S \subseteq N$. The resulting allocation $u_{N}$ is called a Shapley $N T U$ value of $(V, N)$.

Claim 3 (Ex-ante Shapley NTU value). For every $r \in(0,1 / 2)$, the unique Shapley NTU value of the game $\left(V_{r}, N\right)$ is the (ex-ante) utility allocation

$$
\left(U_{1}, U_{2}, U_{3}\right)=(32,32,32) \text {. }
$$

Like the M-value, the Shapley NTU value is independent of $r$. Moreover, it treats all players symmetrically and ignores the fact that coalitions $\{1,3\}$ and $\{2,3\}$ cannot agree on an equitable distribution of the gains.

Definition 4 (Harsanyi NTU value). A payoff configuration $u=\left(u_{S}\right)_{S \subseteq N}$, with $u_{S} \in V(S)$, is a Harsanyi NTU solution of a game $(V, N)$ if there exists a vector of strictly positive utility weights $\lambda=\left(\lambda_{i}\right)_{i \in N}$ such that:

$$
\begin{gathered}
u_{S} \in \partial V(S), \quad \forall S \subseteq N, \\
\sum_{i \in N} \lambda_{i} u_{N}^{i} \geq \sum_{i \in N} \lambda_{i} v^{i}, \quad \forall v \in V(N), \\
\lambda_{i}\left(u_{S}^{i}-u_{S \backslash j}^{i}\right)=\lambda_{j}\left(u_{S}^{j}-u_{S \backslash i}^{j}\right), \quad \forall i, j \in S, \forall S \subseteq N,
\end{gathered}
$$

where $\partial V(S)$ denotes the (Pareto efficient) boundary of $V(S)$. The resulting allocation $u_{N}$ is called a Harsanyi $N T U$ value of $(V, N)$.

Claim 4 (Ex-ante Harsanyi NTU value). For a given $r \in(0,1 / 2)$, the unique Harsanyi NTU value of the game $\left(V_{r}, N\right)$ is the (ex-ante) utility allocation

$$
\left(U_{1}, U_{2}, U_{3}\right)=\left(1-\frac{22 r}{36-33 r}\right) u_{12}+\frac{11 r}{36-33 r} u_{13}+\frac{11 r}{36-33 r} u_{23} .
$$

For every $r$, the weight of the outcome $u_{12}$ of coalition $\{1,2\}$ is the largest. Furthermore, it increases to 1 as $r$ decreases to 0 ; thus the probability of player 3 getting into a coalition converges to 0 . Therefore, the Harsanyi NTU value prescribes an outcome that better captures the lack of transferable utility in this game. 


\section{CONCLUDING REMARKS}

We have analyzed a simple example of an NTU game with incomplete information where the $\mathrm{M}$-value and the $\mathrm{S}$-value yield different outcomes. The computations leading to these outcomes show that the difference between them derives from the way each value measures the strength of intermediate coalitions: while the M-value only reflects the total gains from cooperation, the S-value recognizes, in addition, the restrictions that players face when sharing such proceeds. Similar conclusions are also obtained for the Shapley and Harsanyi NTU values when contracting takes place at the ex-ante stage. We hope that the analysis here will shed further light on the interpretation of the values of cooperative games with incomplete information.

\section{References}

de Clippel, G. (2005). Values for cooperative games with incomplete information: An eloquent example. Games and Economic Behavior, 53, 73-82.

Myerson, R. (1984). Cooperative games with incomplete information. International Journal of Game Theory, 13, 69-96.

Myerson, R. (2007). Virtual utility and the core for games with incomplete information. Journal of Economic Theory, 136, 260-285.

Roth, A. (1980). Values for games without side-payments: Some difficulties with current concepts. Econometrica, 48, 457-465.

Salamanca, A. (2019). A generalization of the Harsanyi NTU value to games with incomplete information. International Journal of Game Theory. 